\title{
Analysis of a TDMA MAC Protocol for Wireless Ad Hoc Networks under Multipath Fading Channels $^{\star}$
}

\author{
José Ramón Gállego, María Canales, Ángela Hernández-Solana, and Antonio \\ Valdovinos
}

Institute of Engineering in Aragón, I3A, University of Zaragoza.

C\ María de Luna, 3, 50.018, Zaragoza (Spain).

jrgalleg@unizar.es, mcanales@unizar.es, anhersol@unizar.es, toni@unizar.es

\begin{abstract}
The behavior of MAC protocols for wireless ad hoc networks is conditioned by multipath fading, especially with regard to the links stability and the differences between channel errors and collisions due to interference. In this paper, we evaluate the impact of multipath fading on the performance of a TDMA proposal designed to provide QoS in wireless ad hoc networks. It lies in a frame subdivision that consists of a broadcast control phase and an information phase. We propose modifications to guarantee the reliability and efficiency of both broadcast control and data services.
\end{abstract}

\section{Introduction}

QoS provision in wireless ad hoc networks requires the support of efficient MAC protocols to solve the tradeoff between resource reservation and channel utilization. In this context, several synchronous approaches have been proposed in order to provide an efficient TDMA access capable of providing resource reservation $[1-5]$. The basic idea behind most of them lies in a reservation cycle with a given number of control mini-slots that allows a node to reserve a conflict-free information slot [1-4]. ADHOC MAC [5] is a proposal that does not require a reservation cycle based on mini-slots to provide access to a broadcast or point-topoint TDMA slot. Using slots of the same size in the whole frame partially alleviates the complexity of the synchronization. It implements a distributed access technique capable of establishing a reliable single-hop Basic broadcast Channel $(\mathrm{BCH})$ for each active terminal. Each $\mathrm{BCH}$ carries signaling information that distributes layer-two connectivity information to all the terminals. Upon the basis of the ADHOC MAC protocol, we have proposed a MAC frame structure that consists of two subframes: A control subframe where terminals contend for a $\mathrm{BCH}$ in order to access the system and a data subframe where active terminals can allocate data communications in a contention-free situation [6].

\footnotetext{
* This work was financed by the Spanish Government (Project TEC2004-04529/TCM from MEC and FEDER), Gobierno de Aragón for WALQA Technology Park and the European IST Project PULSERS Phase II (IST - 027142)
}

Please use the following format when citing this chapter:

Ramón Gállego, J., Canales, M., Hernández-Solana, Á., Valdovinos, A., 2007, in IFIP International Federation for Information Processing, Volume 245, Personal Wireless Communications, eds. Simak, B., Bestak, R., Kozowska, E., (Boston: Springer), pp. 13-24. 
In the evaluation of MAC protocols for wireless ad hoc networks it is often assumed that transmissions that are not sensed do not contribute to the total level of interference a terminal suffers (Protocol Model - [7]). Under these conditions, totally collision-free transmissions are possible with the appropriate MAC signaling. However, when the actual interference produced by all the transmitting terminals is taken into account (Physical Model - [7]), some assumptions carried out under the Protocol Model are no longer valid and collisions can still occur. Moreover, the impact of multipath fading hardly ever is taken into account [8], but it highly conditions the behavior of MAC protocols, especially with regard to the links stability and the differences between errors due to channel conditions and collisions due to interference.

In this paper, we analyze the impact of multipath fading on the performance of the proposed MAC structure. We propose solutions to guarantee the reliability of the protocol in this scenario, including mechanisms to provide link stability and power control procedures that highly increase the spatial reuse. The remaining of the paper is organized as follows. In Section 2 we provide an overview of the considered MAC structure. In Section 3 we show the effect of fading in the protocol operation and the required modifications. In Section 4, simulations results of the proposal are presented. Finally, in Section 5 some conclusions are provided.

\section{MAC Protocol Structure and Operation}

$\mathrm{ADHOC}$ MAC operates with a time slotted structure, where slots are grouped into virtual frames $(V F)$ of length $N$, and no frame alignment is needed. For a correct operation, the $\mathrm{ADHOC} \mathrm{MAC}$ needs that each active terminal has assigned a Basic CHannel (BCH), corresponding to a slot in the $V F$, which is a reliable single hop broadcast channel.

In the $\mathrm{BCH}$, each terminal broadcasts information about the status of the channel it perceives. The BCH contains a control field, the Frame Information (FI). The FI is an $N$-elements vector specifying the status of the $N$ slots preceding the transmission of the terminal itself. The slot status can be either BUSY if a packet has been correctly received or transmitted by the terminal or FREE otherwise. In the case of a BUSY slot, the FI also contains the identity of the transmitting terminal. Based on the received FIs, each terminal marks a slot, say slot $k$, either as RESERVED or AVAILABLE. It is RESERVED if slot $k-N$ is coded as BUSY in at least one of the FIs received in the slots from $k-N$ to $k-1$, and AVAILABLE, otherwise. If a slot is AVAILABLE, it can be used for new access attempts. Upon accessing an AVAILABLE slot, terminal $j$ will recognize, after $N$ slots (a frame), its transmission either successful, if the slot is coded as 'BUSY by terminal $j$ ' in all the received FIs, or failed, otherwise.

Once a terminal has acquired a $\mathrm{BCH}$, it can establish additional data communications with all its neighbors by exploiting this distributed signaling provided by the FIs. In order to efficiently manage the available resources we consider a frame subdivision into $N_{B C H}$ slots for new accesses and $N_{D a t a}$ slots for data 
links among the active terminals. In [9] we propose an adaptive scheme for this subdivision, but in the remaining of the paper, we will consider a static subdivision. In order to establish point-to-point data communications in the data subframe avoiding the exposed terminal problem, each entry of the FI encloses a Point-To-Point (PTP) flag. A terminal sets the PTP flag of a given slot ON if the packet received in the slot is a broadcast one or the terminal itself is the destination. A point-to-point communication can use all the AVAILABLE slots. Further on, even some RESERVED slots can be used if it is satisfied that the PTP flag is signaled OFF in all the received FIs and the FI received from the destination terminal signals the slot as FREE. The whole set of slots that a terminal can select for PTP connections to a given destination will be referred as AVAILABLE PTP slots for that destination.

When dealing with multimedia applications, in response to the demanded QoS, the MAC level must efficiently allocate resources for several differentiated services. To this purpose, we have proposed a reservation scheme [6] to handle the access to the $N_{\text {Data }}$ slots by exploiting the in-band signaling provided by the $\mathrm{BCH}$ that can provide user differentiation through the use of priorities. The basis of this strategy (Book In Advance Scheme - BIAS) relies on the use of the $\mathrm{BCH}$ capabilities to signal the request before the access, in such a way that collisions can be theoretically avoided. The details can be found in [6].

\section{Operation under Fading Channels}

The features of the described protocol guarantee under a simplified Protocol Model that collisions cannot occur once a slot is reserved. However, considering a Physical Model, where the interference produced by all the transmitting terminals is taken into account, a transmission is only considered successful if (1) is satisfied:

$$
S I R_{r x, i, j}^{k}=\frac{P_{t x, i}^{k} \cdot h_{i, j}}{\sum_{\substack{n \in N_{t x}^{k} \\ n \neq i}} P_{t x, n}^{k} \cdot h_{n, j}+P_{n}}>S I R_{t h}
$$

where $S I R_{r x, i, j}^{k}$ is the $S I R$ (Signal to Interference Ratio) received by terminal $j$ from terminal $i$ in slot $k, P_{t x, i}^{k}$ is the power transmitted by user $i$ in slot $k$, $h_{i, j}$ represents the propagation channel between users $i$ and $j, N_{t x}^{k}$ is the set of transmitting terminals in slot $k, P_{n}$ is the thermal noise and $S I R_{t h}$ is the minimum required $S I R$ to decode the information.

In addition to the path loss, dependent on the distance between transmitter and receiver $\left(L_{i, j}\right)$, the propagation channel $h_{i, j}$ includes two random variations in a mobile environment:

- Slow fading or shadowing, due to the terrain variability and often modeled through a lognormal random variable added to the path loss. 
- Fast fading due to multipath propagation, characterized by a Rayleigh distribution when there is not line of sight and with a spectral variation measured through the Doppler spectrum.

Thus, the collision-free property of the reserved slots, theoretically provided in an ideal scenario, cannot be ensured any more due to both channel variations in the received signal and the interference of distant nodes, that can make the received $S I R$ go down the $S I R_{t h}$ in a reserved slot. In order to identify the slots where some power can be sensed, although the terminal is not capable of decoding any information, we include in the FI an additional slot, DIRTY. A terminal cannot transmit in a slot that the potential receiver signals as DIRTY, assuming that the reservation may fail due to interference.

\subsection{Solutions for the broadcast control service}

The maintenance of the $\mathrm{BCH}$ in the $\mathrm{ADHOC} \mathrm{MAC}$ protocol requires the transmitting terminal $i$ to receive the acknowledgment (slot 'BUSY by terminal $i$ ') in all the received FIs from its neighbors. According to this constraint, errors due to channel conditions can induce a high variability in the network activity as a consequence of nodes trying to reallocate a $\mathrm{BCH}$. The control information carried in the $\mathrm{BCH}$ is the basis to perform an appropriate resource allocation and to maintain updated connectivity information, thus this variability makes it more difficult to perform the resource management.

In order to guarantee the correct operation of the broadcast service and to properly choose the nodes suitable to establish reliable point-to-point data links, a set of stable neighbors must be defined. With this purpose, two main problems must be solved. First of all, a criterion to consider a terminal as a stable neighbor is required. Then, when errors in the received packets occur, a mechanism to differentiate a collision due to an interference increase from adverse channel conditions must be provided.

\section{Definition of stable neighbors}

A stable neighbor should guarantee a packet error rate in the communications below certain threshold. In order to fulfill this requirement, the transmissions must be provided with a security margin to overcome the signal and interference variations. An additional threshold $P_{r x-\min }$ is used to verify the reliability of a link. This value is considered as the minimum received power to provide a margin of $\Delta S I R \mathrm{~dB}$ over $S I R_{t h}$ in the absence of any interference. Since this $\Delta S I R$ margin must absorb signal variations due to channel conditions with regard to $\overline{P_{r x}}$ (mean value of the received power), this value has to be known to determine the reliability of a neighbor. Thus, before considering a terminal as a stable neighbor, the received power in the $\mathrm{BCH}$ must be averaged for a given number of frames. With this purpose, we consider a first order IIR filter:

$$
\overline{P_{r x}(n)}=\alpha \cdot \overline{P_{r x}(n-1)}+(1-\alpha) \cdot P_{r x}(n)
$$


where $\overline{P_{r x}(n)}$ is the mean signal power calculated in frame $n, P_{r x}(n)$ the power received in frame $n$ and $\alpha$ the parameter that determines the tradeoff between filtering of fast fading and tracking of slow variations in the mean level of the received signal. The information about the useful received signal $P_{r x}$ can be obtained from the estimated $S I R[10]$ and the total received power.

Thus, we consider that a terminal is a stable neighbor when $\overline{P_{r x}(n)}>P_{r x, \text { min }}$ in the $\mathrm{BCH}$. Since the estimation of the received power varies around the real mean level, those nodes with a mean power around $P_{r x, \text { min }}$ can enter in an oscillating dynamic 'stable-non stable' that can complicate the establishment of links with them. In order to avoid this situation, an hysteresis mechanism is incorporated, in such a way that once $\overline{P_{r x}(n)}>P_{r x, \min }$ and the terminal is considered as a stable neighbor, the estimation must be $\Delta P_{\text {est }} \mathrm{dB}$ lower than $P_{r x, \text { min }}$ to stop being a stable neighbor $\left(\overline{P_{r x}(n)}<P_{r x, \text { min }}-\Delta P_{e s t}\right)$.

\section{Collision Detection}

Fading effects can lead to receive a packet from an stable neighbor with $S I R<S I R_{t h}$ even when the total level of interference is zero. According to the rules of the protocol, an error is always understood as a collision and a rescheduling of the $\mathrm{BCH}$ in a different slot is needed. However, when errors are caused by fading, the reallocation of the transmission does not solve the problem, since while the fading lasts, the quality of the received signal is low in any slot. In fact, continuous reschedulings due to channel errors lead to an important increase in the network instability. As a consequence, collision detection cannot be carried out through instantaneous values, but mean $S I R$ values. We consider that a terminal suffers a collision if the average $S I R$ goes down while the average received power keeps constant, i.e., if there is an increase in the mean level of interference.

The mean value of the received signal to interference ratio, $\overline{S I R}$, is obtained with the same filter as (2), applied over the estimated $S I R$ values on a frame by frame basis. Then, when an error in the reception of the BCH from stable neighbor occurs (i.e., $\overline{P_{r x}}>P_{r x, m i n}$ ), it is checked whether $\overline{S I R}<S I R_{\text {collision }}$. Only if both conditions are fulfilled, the receiver will treat this error as a collision and will report it to the transmitter so that this one reallocates the transmission, as it is shown in figure 1. In order to differentiate a transmission error from the report of a collision (DIRTY status), an additional status in the FI is required in order to notify a negative ACK that does not require a slot reallocation. We name that status as ERROR, in such a way that terminal $i$ will maintain its slot $\mathrm{BCH}$ as long as it observes the slot as 'BUSY or ERROR by terminal i' in all the received FIs from its stable neighbors.

The election of the specific value for $S I R_{\text {collision }}$ implies a tradeoff in the protocol design. High values, near the $S I R$ that all the stable neighbors must guarantee when there is no interference $\left(S I R_{t h}+\Delta S I R=P_{r x, \min }-P_{n}\right.$ in $\left.\mathrm{dB}\right)$ lead to mistakes between channel errors and collisions. On the other hand, low values near $S I R_{t h}$ can disguise the existence of a collision, leading to keep on transmitting in a slot where the interference level has notably grown, with the subsequent increase in the packet error rate. 

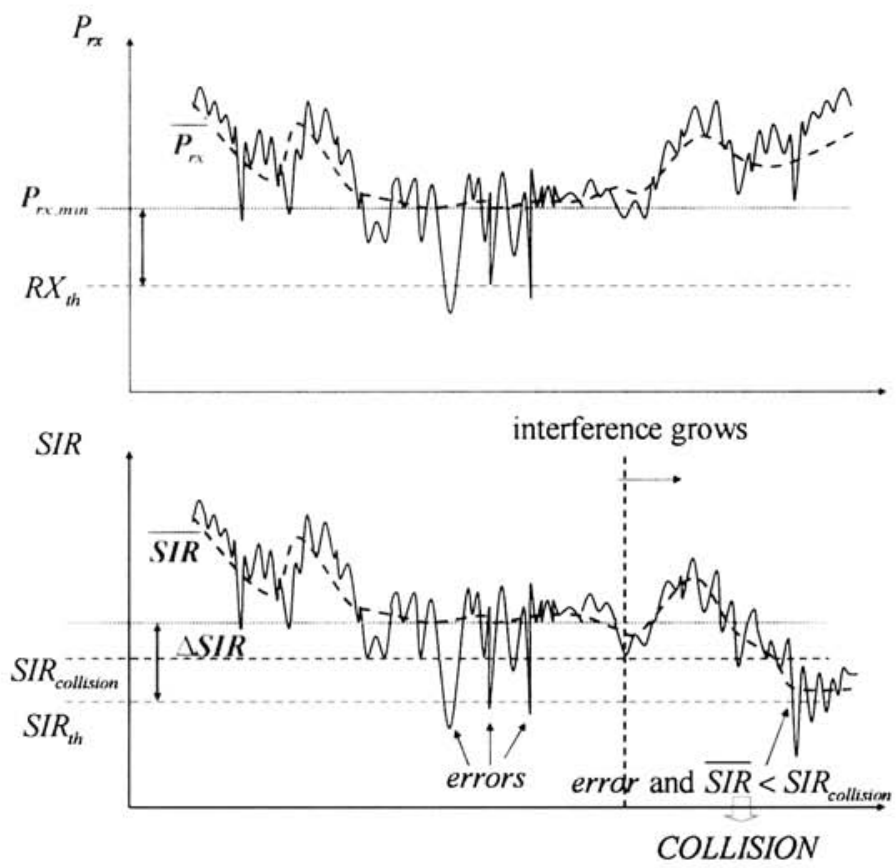

Fig. 1. Example of collision detection under fading.

\subsection{Solutions for the point to point data service}

We consider that point to point data communications can only be established between stable neighbors in order to guarantee the reliability of the dispatched connections. In these transmissions, differentiation between channel errors and collisions is also required. Therefore, in order to detect a collision in a data slot $k$, a terminal must calculate the average $S I R$ in that slot, $\overline{S I R_{\text {data }, k}}$, in the same way as with the $\mathrm{BCH}$. Thus, when there is an error in a data packet and $\overline{S I R_{\text {data }, k}}<S I R_{\text {collision }}$ a collision will be reported (DIRTY in the FI), while if $\overline{S I R_{\text {data }, k}}>S I R_{\text {collision }}$ only a negative acknowledgment will be sent (ERROR in the FI).

Power control has been shown to increase spatial reuse leading to an improvement in the network capacity [7]. The signaling information distributed by all the neighbors in their FI at a fixed transmission power, $P_{t x-\max }$, supplies the basis to implement power control procedures in our proposed MAC structure [11]. This power control mechanism is based on estimating the propagation channel from the received power in the $\mathrm{BCH}$ of the destination terminal and, assuming symmetrical links, using this estimation to calculate the required transmission power. Since both terminals transmit in the same carrier frequency and with the same bandwidth, we can consider the bidirectionality to be valid. However, under time varying multipath channels, this scheme presents a main problem, consisting of the validity of the estimation carried out in a specific time to be 
applied in a later time. As terminal speeds grows, the previous estimation is less correlated with the actual channel, making unviable this mechanism. Therefore, the chosen alternative to solve these problems lies in carrying out the power control according to the mean value of the channel loss, in such a way that the transmission power must provide an additional margin to protect the signal against the fast fading. Moreover, this mean value can be directly obtained from the mean received power, $\overline{P_{r x}}$, already calculated for the management of the stable neighbors.

A slot can be used to establish a connection if the receiver signals the slot as FREE and there is not any receiving terminal in the surroundings of the transmitter. The adjustment of the transmission power allows to reduce the interference over distant connections, thus reducing the number of DIRTY slots, and consequently increasing the number of accessible FREE slots, which can lead to an improvement in the network capacity.

We assume that terminal $i$ wants to establish a connection with terminal $j$ in slot $k$, signaled as FREE by $j$. According to the estimated information about the channel loss, $\overline{h_{i, j}}$, terminal $i$ can set its transmission power $P_{t x, i}^{k}$, on a frame by frame basis, to provide a target $S I R\left(S I R_{t a r}\right)$, which must guarantee a margin to overcome variations in the received signal and in the interference level:

$$
P_{t x, i}^{k}= \begin{cases}\frac{S I R_{t a r} \cdot}{h_{i, j}} \cdot P_{0} & \text { if } P_{t x, i}^{k} \leq P_{t x-\max }, \\ P_{t x-\max } & \text { if } P_{t x, i}^{k}>P_{t x-\max } .\end{cases}
$$

where the mean channel loss from $i$ to $j, \overline{h_{i, j}}$ is estimated by means of the mean received power from $j$ to $i, \overline{P_{r x, j, i}}: \overline{h_{i, j}}=\overline{h_{j, i}}=\frac{\overline{P_{r x, j, i}}}{P_{t x-\text { max }}}$.

A more sophisticated procedure to increase the spatial reuse can be carried out by including in the FI additional information about the mean level of interference that a terminal estimates in each slot, $\hat{P}_{i n t}^{k}$, at the expense of increasing the control information sent in the $\mathrm{BCH}$. In that case, the transmitter can access slot $k$ regardless of being DIRTY or BUSY if:

$$
P_{t x, i}^{k}=\frac{S I R_{t a r} \cdot\left(P_{n}+\overline{\hat{P}_{i n t}^{k}}\right)}{\overline{h_{i, j}}} \leq P_{t x-\max }
$$

\section{Performance Evaluation}

The impact of multipath fading in the proposed MAC architecture has been evaluated by means of simulation. With this purpose, we have built up an event driven simulator in $\mathrm{C}++$ which implements all the required functionalities. 400 terminals are randomly positioned within a square area of $1 \mathrm{Km}^{2}$. The considered mobility pattern is based on the well-known Random Way-Point (RWP) mobility model. The RWP is modified in such a way that each new destination of the model is not completely random, but it must not suppose a change in the current direction of the terminal higher that some specific angle $(\pi / 12$ in 
the simulations). Each terminal generates PTP data communications according to a Poisson process with intensity X (PTP connections/s). The source of each point-to-point communication is randomly chosen among the users with an active $\mathrm{BCH}$, while the destination is randomly chosen among the source's neighbors. The duration of each communication is exponentially distributed with mean $\mathrm{D}=50$ (frames). Likewise, each connection generates 1 packet per frame (CBR). X and $\mathrm{D}$ define the point-to-point offered traffic by each terminal. Each frame consists of 75 slots $\left(N_{B C H}=50, N_{\text {Data }}=25\right)$, transmitted each $20 \mathrm{~ms}$ at $11 \mathrm{Mbps} . S I R_{t h}$ is set to $5 \mathrm{~dB}, P_{n}$ is $-103 \mathrm{dBm}$ and $C S_{t h}$ (Carrier Sense Threshold) is $-102 \mathrm{dBm}$. Path loss is $L=-128.1-37.6 \cdot \log _{10}(d) \mathrm{dB}$ (d in $\left.\mathrm{Km}\right)$. The maximum transmission power, $P_{t x-\max }$, is set to $-7.5 \mathrm{dBm}$, which provides a maximum transmission range of $100 \mathrm{~m}$. Log-normally distributed shadowing with standard deviation of $6 \mathrm{~dB}$ is also included. Two multi-path fading environments (pedestrian and vehicular - [12]) have been considered.

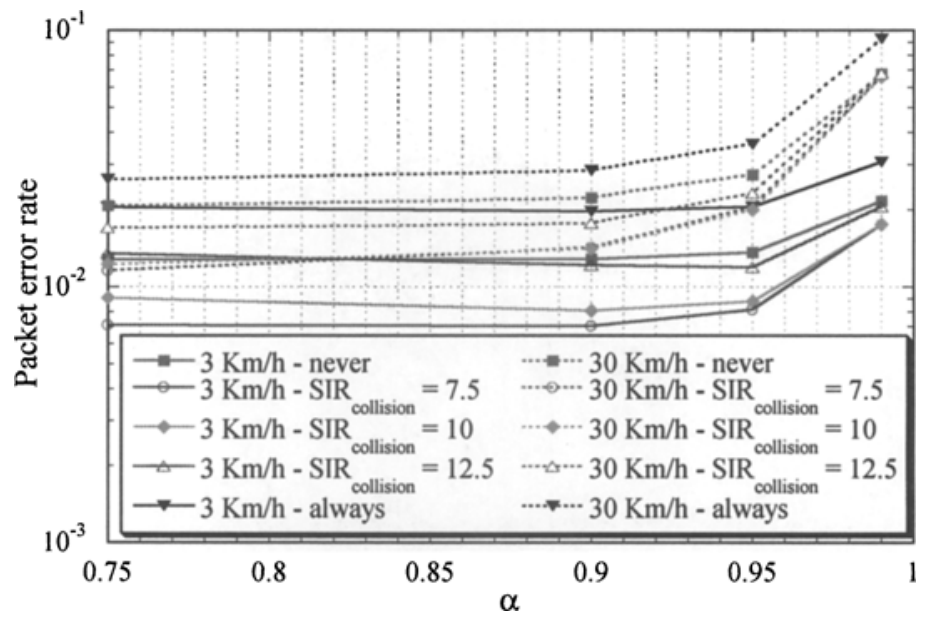

Fig. 2. Packet error rate for the stable neighbors in the broadcast service. $\Delta S I R=10$ dB. Different values of $S I R_{\text {collision }}$.

Figure 2 shows the packet error rate for the broadcast service according to $\alpha$ (filtering of the received power) for different values of $S I R_{\text {collision }}$ with a margin against fading and interference of $10 \mathrm{~dB}(\Delta S I R)$ and $\Delta P_{e s t}=3 \mathrm{~dB}$. This error rate is defined as the ratio between wrong and total receptions in stable neighbors. Two extreme situations regarding collision detection are included: never detecting collision, regardless of the estimated $S I R$ and always that an error is detected reporting a collision. High values for $\alpha(0.99)$, lead to an important increase in the error rate: This rate is related to the errors in stable neighbors, whose definition is given by the filtering of the received power. With high values for $\alpha$, the estimated average power $\overline{P_{r x}}$, not only filters fast fading, but also the changes in channel loss due to terminals movement or shadowing, 
in such a way that a neighbor can be considered as stable despite not providing the required margin. With regard to $S I R_{\text {collision }}$, the extreme cases (always and never) provide the worst performance. If all errors are treated as collisions, the frequent reallocations of the $\mathrm{BCH}$ lead to an scenario of permanent contention for the resources, which increases access collisions and as a result, the error rate. When collisions are not detected, transmissions in slots suffering high levels of interference can persist, also increasing the error rate. In this scenario, a $S I R_{\text {collision }}$ of 7.5 or $10 \mathrm{~dB}$ provides a good tradeoff for both 3 and $30 \mathrm{~km} / \mathrm{h}$.

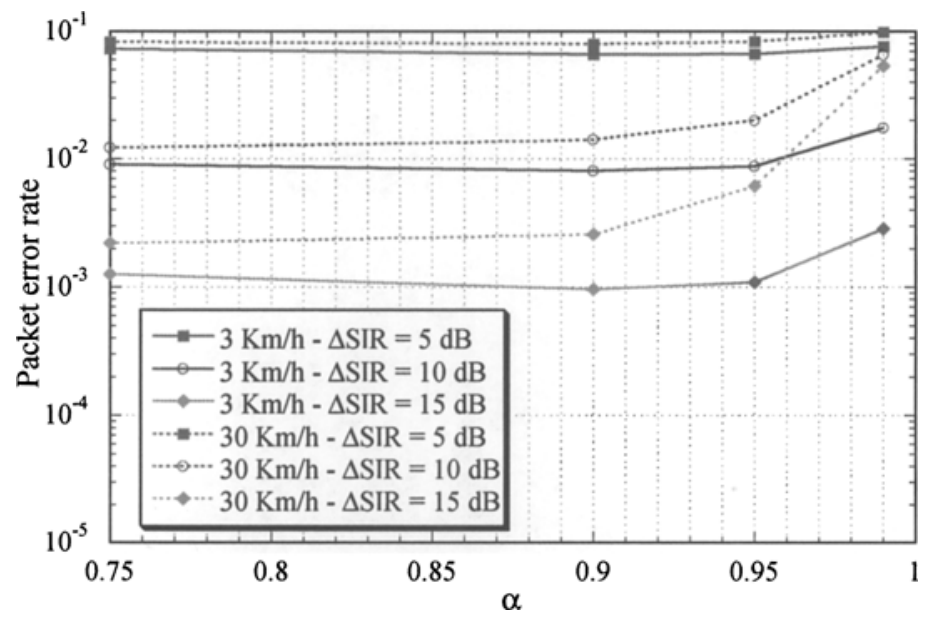

Fig. 3. Packet error rate for the stable neighbors in the broadcast service. Different values of $\triangle S I R$.

Table 1. Mean duration of stable links (sec).

\begin{tabular}{|c|c|c|}
\hline \multirow{2}{*}{$\alpha$} & \multicolumn{2}{|c|}{ Speed } \\
\cline { 2 - 3 } & $3 \mathrm{Km} / \mathrm{h}$ & $30 \mathrm{Km} / \mathrm{h}$ \\
\hline 0.75 & 3.2 & 3.2 \\
\hline 0.9 & 9.6 & 6.1 \\
\hline 0.95 & 16.3 & 7.6 \\
\hline 0.99 & 32.1 & 13.8 \\
\hline
\end{tabular}

With an analogous analysis for $\triangle S I R$ of 5 and $15 \mathrm{~dB}, S I R_{\text {collision }}$ values of 7.5 and $12.5 \mathrm{~dB}$ also provide a good tradeoff with regard to the collision detection. Figure 3 shows similar results, in this case for different values of $\Delta S I R(5,10$ and $15 \mathrm{~dB})$, fixing the $S I R_{\text {collision }}$ that provides best performance in each case $(7.5,10$ and $12.5 \mathrm{~dB})$. Logically, the higher $\Delta S I R$, the lower the 
error rate. However, an increase in $\Delta S I R$ implies a reduction in the effective coverage area, and consequently, in the number of terminals allowed to establish data communications. Thus, excessively high values for $\Delta S I R$ can lead to a higher probability of unconnected network. In the evaluation of point to point data services, a $\triangle S I R=10 \mathrm{~dB}$ has been chosen as a tradeoff between effective coverage area and reliability (error rate).

Regarding the election of $\alpha$, in addition to the error rate, the capacity of reliably determining the set of stable neighbors is also important in order to establish efficient data communications. Table 1 shows mean duration of stable links for different values of $\alpha$. The estimation of $\overline{P_{r x}}$ is more resistant against channel variations as $\alpha$ grows, and therefore, the duration of stable links is higher. However, as it is seen in figures 2 and $3, \alpha=0.99$ or even 0.95 for high speeds lead to wrong estimations in the stable links that make error rate grow. Therefore, we choose $\alpha=0.9$ in order to evaluate data services.

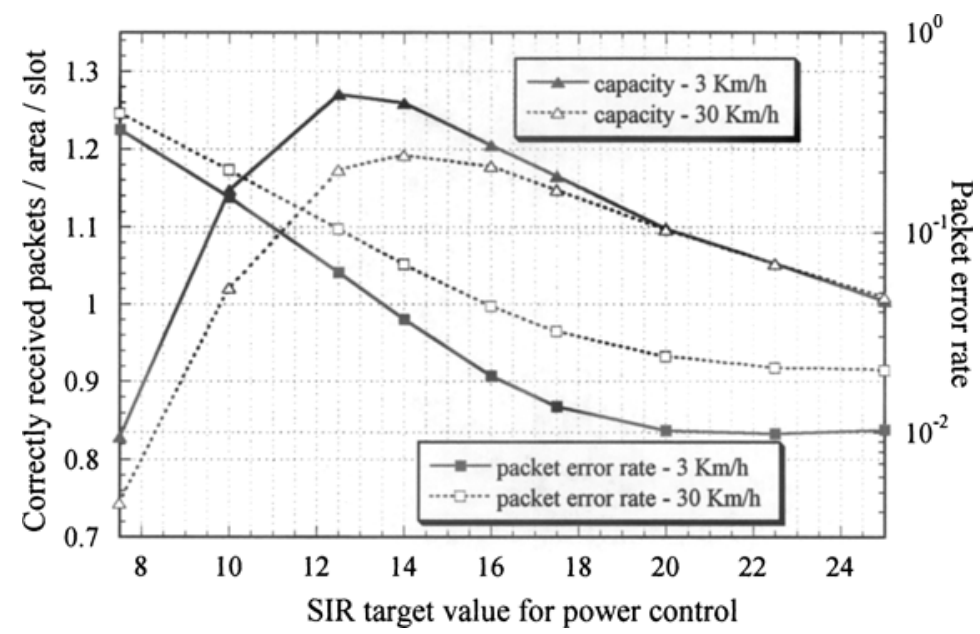

Fig. 4. Power control behavior for different $S I R_{t a r}$ values. Mean PTP offered traffic $=20$ connections $/$ terminal.

Figure 4 shows the capacity and the error rate for point to point data services obtained for different $S I R_{t a r}$ values when only FREE slots are used to access. The capacity is normalized to the number of data slots $\left(N_{\text {Data }}\right)$ and the maximum coverage area (range: $100 \mathrm{~m}$ ). Power control requires a tradeoff between reliability and capacity. The error rate notably decreases as the $S I R_{t a r}$ grows, specially up to 18 or $20 \mathrm{~dB}$, since the available margin is higher. However, the maximum capacity is obtained around $S I R_{t a r}=12 \mathrm{~dB}$, and decreases for higher vales since the spatial reuse diminishes. We consider a tradeoff value of $17.5 \mathrm{~dB}$.

Figure 5 compares the performance without power control with two different alternatives: basic mode with access only in the FREE slots (Basic) and access 
also in the BUSY or DIRTY slots according to the interference information distributed in the FIs (Interference-Aware), all of them with a mean terminal speed of $30 \mathrm{Km} / \mathrm{h}$. Power control techniques involve a slight increase in the packet error rate, since the margin against fading and interference is reduced with regard to the situation where each terminal transmits at the maximum available power. However, in spite of this fact, the number of connections correctly dispatched is significantly higher thanks to the increase in the spatial reuse, specially when interference information $\left(\hat{P}_{i n t}^{k}\right)$ is used.

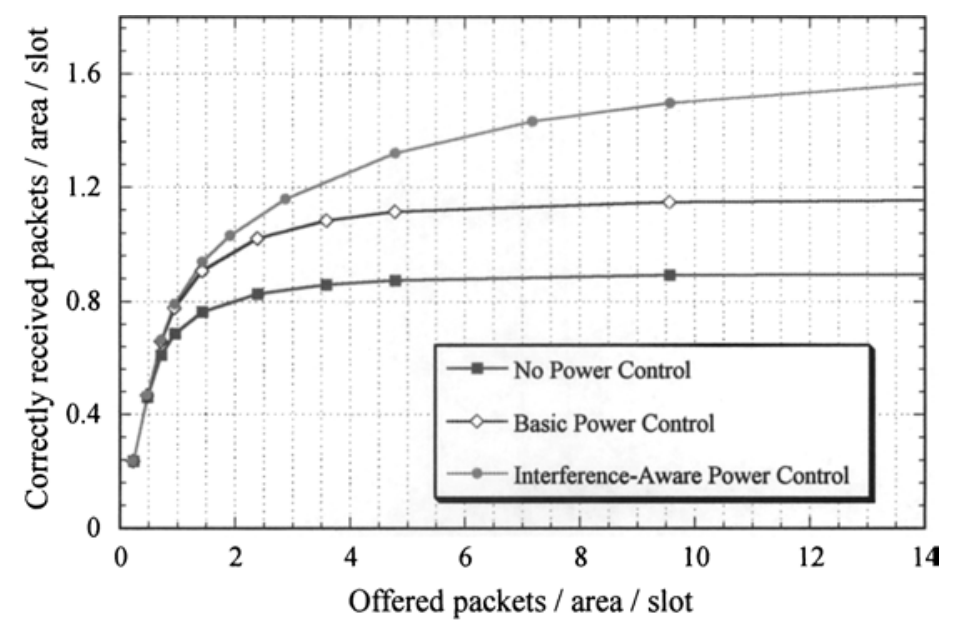

(a) Capacity

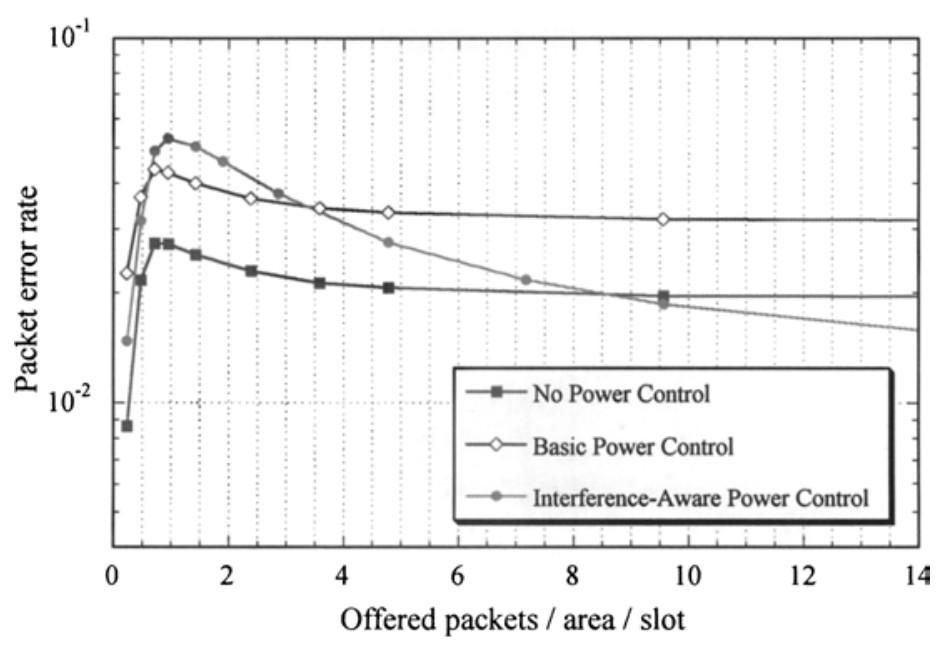

(b) Packet error rate

Fig. 5. Capacity and packet error rate for point-to-point data services with different strategies. $S I R_{t a r}=17.5 \mathrm{~dB}$ for power control. 


\section{Conclusions}

In this paper, we have analyzed the impact of multipath fading on the performance of a MAC proposal for QoS provisioning in wireless ad hoc networks based on a TDMA access. We have proposed solutions to guarantee the reliability and stability of both broadcast control and data services, thanks to the definition of a set of stable neighbors based on the average received power. Differentiation between errors due to channel conditions from collisions due to an interference increase has been provided. Finally, the validity of power control procedures to increase the network capacity has been shown.

\section{References}

1. Tang, Z., Garca-Luna-Aceves, J.J.: A protocol for topology-dependent transmission scheduling in wireless networks. In: Proc. of IEEE WCNC'99. Volume 3., New Orleans, USA (1999) 1333-1337

2. Zhu, C., Corson, M.S.: A Five-Phase Reservation Protocol (FPRP) for mobile ad hoc networks. Wireless Networks (WINET) 7(4) (2001) 371-384

3. Fang, J.C., Kondylis, G.D.: A synchronous, reservation based medium access control protocol for multihop wireless networks. In: Proceedings of IEEE WCNC'03. Volume 2., New Orleans, USA (March 2003) 994-998

4. Ahn, C.W., Kang, C.G., Cho, Y.Z.: Soft reservation multiple access with priority assignment (SRMA/PA): A distributed MAC protocol for QoS-guaranteed integrated services in mobile ad-hoc networks. IEICE Transactions on Communications E86-B(1) (January 2003) 50-59

5. Borgonovo, F., Capone, A., Cesana, M., Fratta, L.: ADHOC MAC: a new MAC architecture for ad hoc networks providing efficient and reliable point-to-point and broadcast services. Wireless Networks (WINET) 10(4) (2004) 359-366

6. Gállego, J.R., Canales, M., Hernández-Solana, A., Campelli, L., Cesana, M., Valdovinos, A.: Performance evaluation of point-to-point scheduling strategies for the ADHOC MAC protocol. In: Proc. of WPMC'05, Aalborg, Denmark (2005) 1380-1384

7. Gupta, P., Kumar, P.R.: The capacity of wireless networks. IEEE Transactions on Information Theory $46(2)$ (2000) 388-404

8. Mullen, J., Huang, H.: Impact of multipath fading in wireless ad hoc networks. In: Proc. of ACM PE-WASUN'05, Montreal, Canada (2005) 181-188

9. Gállego, J.R., Campelli, L., Cesana, M., Capone, A., Borgonovo, F., HernándezSolana, A., Canales, M., Valdovinos, A.: Efficient bandwidth allocation for basic broadcast and point-to-point services in the ADHOC MAC protocol. In: Proc. of IFIP PWC'05, Colmar, France (2005) 87-98

10. Lau, F., Tam, W.: Novel SIR-estimation-based power control in a CDMA mobile radio system under multipath environment. IEEE Transactions on Vehicular Technology 50(1) (2001) 314-320

11. Gállego, J.R., Canales, M., Hernández-Solana, A., Valdovinos, A.: Performance analysis of an interference-aware MAC protocol with power control for wireless ad hoc networks. In: Proc. of IEEE PIMRC'06, Helsinki, Finland (2006)

12. 3GPP: Universal mobile telecommunications system (umts); selection procedures for the choice of radio transmission technologies of the umts (umts 30.03 version 3.2.0). Technical Report 101112 V3.2.0, 3GPP (1998-04) 